\title{
Inventario sistemático revisado y actualizado de los moluscos marinos ocurrentes en el Estado de Santa Catarina, Brasil
}

\section{A. Ignacio Agudo-Padrón}

Projeto “Avulsos Malacológicos - AM,” Caixa Postal (P.O. Box) 010, Centro, Florianópolis-SC (CEP 88010-970). E-mail: ignacioagudo@gmail.com. http://noticiasmalacologicas-am.webnode.pt.

Resumen. Producto de 19 años completos de investigaciones de campo, examen de especímenes depositados em colecciones de museos y estudios referenciales paralelos, el inventário malacológico sistemático marino del Estado de Santa Catarina, Región Subtropical Central Sur del Brasil, es finalmente presentado, comportando un total de 671 espécies e subespécies confirmadas (11 Polyplacophora, 400 Gastropoda, 10 Scaphopoda, 226 Bivalvia y 24 Cephalopoda), distribuídas en 365 gêneros e 152 famílias, correspondientes al 42\% del total estimado para el Brasil en general. De estas apenas dos son contempladas por categorias oficiales de conservación, cuatro constituyen reconocidas formas exóticas invasoras, y al menos otras 10 encuentranse directamente envolvidas en actividades antrópicas de maricultura, pesca y extractivismo.

Palabras Clave: Moluscos marinos, Estado de Santa Catarina, Región sur del Brasil, Inventário de espécies.

Resumo. Inventário sistemático revisado e atualizado dos moluscos marinhos ocorrentes no Estado de Santa Catarina, Brasil. Produto de 19 anos completos de pesquisas em campo, o exame de espécimes depositados em coleções de museus e estudos referenciais paralelos, o inventário sistemático malacológico marinho do Estado de Santa Catarina, Região Subtropical Central Sul do Brasil, finalmente é apresentado, comportando um total de 671 espécies e subespécies confirmadas (11 Polyplacophora, 400 Gastropoda, 10 Scaphopoda, 226 Bivalvia e 24 Cephalopoda), distribuídas em 365 gêneros e 152 famílias, correspondentes a 42\% do total estimado para o Brasil. Destas, apenas duas são contempladas por categorias oficiais de conservação, quatro constituem reconhecidas formas exóticas e ao menos outras 10 encontram-se diretamente envolvidas em atividades antrópicas de maricultura, pesca e extrativismo.

Palavras-Chave. Moluscos marinhos, Estado de Santa Catarina, Região Sul do Brasil, Inventário de espécies.

Abstract. Systematic inventory revised and updated from occurring marine mollusks in the state of Santa Catarina, Brazil.. Product of complete 19 years of field researches, examination of specimens deposited in museum collections and parallel referencial studies, the systematic marine malacological inventory of Santa Catarina's State, Subtropical Central Southern Brazil Region, is finally presented, behaving a total of 671 confirmed species and

Recebido: 13/04/2015

Aceito: 20/06/2015

Publicado: 30/06/2015

Acesso Aberto

Artigo completo

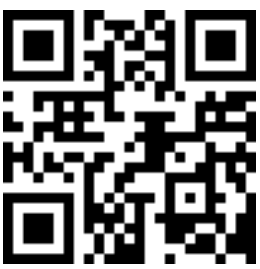


subspecies (11 Polyplacophora, 400 Gastropoda, 10 Scaphopoda, 226 Bivalvia and 24 Cephalopoda), included in 365 genera and 152 families, corresponding to $42 \%$ of the estimate for Brazil in general. Of these only 2 are covered by official conservation categories, 4 are recognized invasive alien forms, and at least 10 are directly envolved in human activities of mariculture, fishing and extrativism.

Keywords. Marine molluscs, Santa Catarina State, Southern Brazil Region, Species inventory.

\section{Introducción}

El estudio y conocimiento de la riqueza/diversidad de moluscos existente en las águas marinas costeras y oceánicas del Estado de Santa Catarina, la porción geopolítica más pequeña y central de la Región Sur del Brasil, así como en otros lugares del mundo, resulta urgente, en vista de las rápidas modificaciones que actualmente sufre su medio ambiente natural, consecuencia de las diversas actividades humanas allí realizadas, creando situaciones de amenaza todavía poco comprendidas regionalmente. Esto se ha visto agravado en algunos casos por deficiencias en el conocimiento taxonómico y general de ciertas espécies (Agudo-Padrón, 2014b: 36), así como también, por uma parte, del rápido y complejo proceso de invasión experimentado por formas exóticas introduzidas - sea por accidente 0 propositalmente (Agudo-Padrón y Bleicker, 2009c; Melo et al., 2010; Agudo-Padrón, 2011a), y por la outra, las contemporâneas actividades antrópicas exploratórias para consumo alimentício, representadas en la práctica por la maricultura, la pesca industrial predadora y el extractivismo mal ejecutado (Agudo-Padrón y Bleicker, 2009b).

Conforme estimativas disponibles en la literatura (Ribeiro-Costa y Marinoni, 2006: 81; Thomé et al., 2010: 7), existen actualmente en el Brasil alrededor de 1.600 (cerca de dos mil) espécies registradas/catalogadas de moluscos marinos. Tales números, sin embargo, todavía son considerados "subestimados" por algunos especialistas, debido entre otros a la sensible falta de mayores estudios aprofundados, así como también a la indisponibilidad representantiva de algunas de esas espécies en colecciones científicas regionales.
Como inicialmente comentado, el territorio subtropical del Estado de Santa Catarina es la porción geo-política mas pequeña y central de la Región Sur del Brasil, geograficamente localizada entre los Estados de Paraná e Rio Grande do Sul, haciendo frontera en su extremo occidental con la República de Argentina (no mas que $1.13 \%$ de la vasta región total del país), entre los paralelos $25^{\circ}-30^{\circ}$ de Latitud S y los meridianos $48^{\circ}-54^{\circ}$ de Longitud $\mathrm{W}$, midiendo sus pontos mas distantes $377 \mathrm{~km}$ en dirección Norte-Sur y 547 km en dirección Este-Oeste). Su borde geográfico oriental, con una extensión de $580 \mathrm{~km}$ de sinuoso y diversificado litoral Atlántico Continental e insular (Figura 1) localizase en amplia zona de transición consecuencia del encuentro/convergencia entre dos importantes corrientes marinas, la del Brasil (caliente Ecuatorial, del Norte) y la de Las Malvinas o Falkland (fría Austral, del Sur), condición esta que en general determina una rica y singular composición heterogénea local, tanto faunística como del medio ambiente. Para una conveniente y completa descripción geográfica, incluyendo mapeamentos de su porción litoral, ver Agudo-Padrón et al. (2009, 2013), Agudo-Padrón (2012, 2015) y Agudo-Padrón y Bleicker (2014: 14, Figura 1).

El conocimiento de la fauna de moluscos de una región es importante sobre varios aspectos, tales como zoológico, biogeográfico, arqueológico, paleontológico, recurso alimentício y conservación ecológica, así como de interes médicosanitário y veterinário (específico para formas continentales terrestres y límnicas/ de agua dulce) en su condición de vectores de enfermedades parasíticas incidentes en seres humanos y animales domésticos (Agudo-Padrón et al., 2013). Entre tanto, falta mucho que añadir todavia para ser 


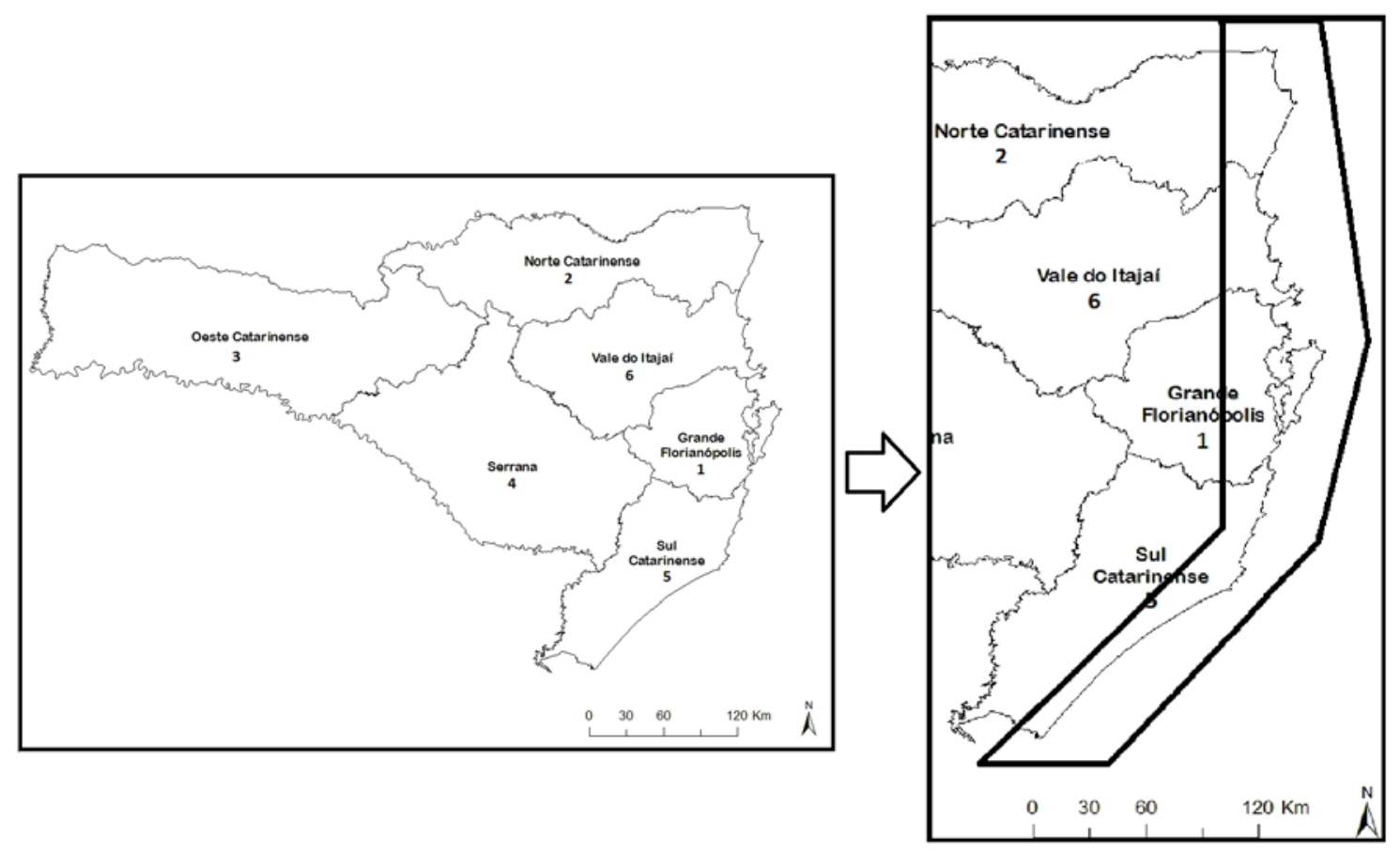

Figura 1. Localización de la área marino-costera atlântica - continental e insular - del Estado de Santa Catarina (destaque), en referencia a su división geo-política territorial, envolviendo las 4 regiones en contacto directo con el mar: (1) Región Grande Florianópolis; (2) Región Norte; (5) Región Sur; y (6) Región Valle del Rio Itajaí.

completado el catálogo general de los moluscos ocurrentes en el vasto território del Brasil (Oliveira y Castro, 1979; Oliveira y Almeida, 2000).

Basado en amplia revisión bibliográfica, desarrollada a partir de los resultados obtenidos en la etapa precedente del inventário (Agudo-Padrón et al., 2009), concentrada en las contribuciones de Agudo-Padrón y Bleicker (2008, 2009a, 2009c, 2011, 2014) y Agudo-Padrón (2011b, 2012, 2014b, 2015), así como en el examen de especímenes depositados en colecciones institucionales, incluyendo aquellas muestras obtenidas en el transcurso de trabajos realizados en campo, la presente contribución finalmente incorpora el registro total de 671 taxa al inventario sistemático de moluscos marinhos del Estado de Santa Catarina (Tabla 1), incluyendo 11 taxa de Polyplacophora, 400 de Gastropoda, 10 de Scaphopoda, 226 de Bivalvia y 24 de Cephalopoda, distribuídas en 365 gêneros y 152 famílias regionalmente conocidas, correspondientes al $42 \%$ del total estimado para el Brasil en general (Ribeiro-Costa y Marinoni, 2006: 81), producto consolidado de casi dos décadas completas de investigación sistemática sostenida iniciada en Marzo de 1996 (Agudo-Padrón, 2012: 15-16), incluyendo mas tres espécies Gastropoda representantes de las Famílias Trochidae Gaza compta Simone \& Cunha, 2006, Rissoidae - Alvania cruzi (Castellanos \& Fernández, 1974), y Buccinidae Gemophos auritulus (Link, 1807), reportadas todas para el Estado en la literatura de reciente producción disponible (Wiggers y Veitenheimer-Mendes, 2009; CdB, 2010).

El ordenamiento taxonómico adoptado, incluyendo el critério sistemático de presentación de las categorías de género, espécie y subespécie dentro de las respectivas famílias, acompaña la propuesta original desarrollada en la etapa inicial del inventário (Agudo-Padrón et al., 2009), revisada, organizada y complementada siguiendo las contribuciones monográficas de García et al. (2008), Rios (2009) y Thomé et al. (2010), principalmente.

Informaciones adicionales acerca del material examinado, tipos depositados en museos y distribución geográfica 
Tabla 1. Total de especies de moluscos marinos/estuarinos y continentales/anfibios ocurrentes en el Estado de Santa Catarina, Región Central Sur del Brasil, contabilizada hasta el mes de Abril de 2015.

\begin{tabular}{|c|c|c|c|}
\hline Classe Considerada & Espécies & Géneros & Familias \\
\hline \multicolumn{4}{|l|}{ Marina/ Estuarina } \\
\hline Polyplacophora & 11 & 5 & 2 \\
\hline Gastropoda & 400 & 213 & 89 \\
\hline Scaphopoda & 10 & 3 & 2 \\
\hline Bivalvia & 226 & 124 & 48 \\
\hline Cephalopoda & 24 & 20 & 13 \\
\hline Total & 671 & 365 & 154 \\
\hline \multicolumn{4}{|c|}{ Continental/Anfíbia } \\
\hline Gastropoda Terrestre & 154 & 68 & 22 \\
\hline Gastropoda Límnica & 40 & 19 & 8 \\
\hline Bivalvia Límnica & 30 & 11 & 5 \\
\hline Total & 224 & 98 & 35 \\
\hline Total General & 895 & 463 & 189 \\
\hline
\end{tabular}

Fuente: Banco de datos referenciales analizados por el autor (1996-2015).

conocida, correspondiente a las especies a seguir relacionadas, pueden ser obtenidas en los trabajos antes comentados.

\section{Inventário sistemático}

Classe Polyplacophora Gray, 1821

Orden Neoloricata Bergenhayn, 1955

Família Ischnochitonidae Dall, 1889

Chaetopleura angulata (Splengler, 1797)

Chaetopleura asperrima (Gould, 1852)

Chaetopleura isabellei (d'Orbigny, 1841)

Chaetopleura sowerbiana (Reeve, 1847)

Ischnochiton hartmeyeri Thiele, 1910

Ischnochiton lopesi Kaas, 1974

Ischnochiton striolatus (Gray, 1828)

Ischnochiton pectinatus (Sowerby, 1840)

Stenoplax purpurascens (C. B. Adams, 1845)

Calloplax janeirensis (Gray, 1828)

Familia Hanleyidae Gray, 1857

Hanleya brachyplax Simone \& Jardim, 2009

Classe Gastropoda Cuvier, 1787

Subclasse Prosobranchia Milne Edwards, 1848

Orden Archaeogastropoda Thiele, 1925

Familia Pleurotomariidae Swainson, 1840

Perotrochus atlanticus Rios \& Matthews, 1968

Familia Fissurellidae Fleming, 1822

Cranopsis (= Puncturella) granulata (Seguenza, 1863)

Diodora cayenensis (Lamarck, 1822)

Diodora dysoni (Reeve, 1850)
Diodora harrassowitzi (Ihering, 1927)

Diodora meta (Ihering, 1927)

Diodora patagonica (d'Orbigny, 1847)

Fissurellidea megatrema d'Orbigny, 1841

Fissurella clenchi Farfante, 1943

Fissurella rosea (Gmelin, 1791)

Lucapinella limatula (Reeve, 1850)

Lucapinella henseli (Martens, 1900)

Familia Acmaeidae Carpenter, 1857

Collisella (= Acmaea) subrugosa (d’Orbigny, 1846)

Familia Trochidae Rafinesque, 1815

Tegula patagonica (d’Orbigny, 1840)

Tegula viridula (Gmelin, 1791)

Calliostoma coppingeri (E. A. Smith, 1880)

Calliostoma jucundum (Gould, 1849)

Calliostoma depictum Dall, 1927

Calliostoma jujubinum (Gmelin, 1791)

Calliostoma adspersum (Philippi, 1851)

Calliostoma axelolssoni Quinn, 1992

Calliostoma echinatum Dall, 1881

Calliostoma militaris ( Ihering, 1907)

Calliostoma barbouri Clench \& Aguayo, 1946

Calliostoma sapidum Dall, 1881

Calliostoma rota Quinn, 1992

Photinula blakei (Clench \& Aguayo, 1938)

Solariella carvalhoi Lopes \& Cardoso, 1958

Halistylus columma Dall, 1890

Gaza compta Simone \& Cunha, 2006

Familia Skeneidae Thiele, 1929

Cyclostremiscus pentagonus (Gabb, 1873)

Cyclostremiscus beauii (Fischer, 1857) 
Familia Turbinidae Rafinesque, 1815

Astraea (= Astralium) latispina (Philippi, 1844)

Astraea tecta (Lightfoot, 1786)

Astraea (= Lithopoma) olfersii (Philippi, 1846)

Astraea phoebia Röding, 1798

Arene microforis (Dall, 1889)

Arene variabilis (Dall, 1889)

Familia Tricoliidae Robertson, 1958

Tricolia (= Eulithidium) affinis (= affine) (C. B. Adams, 1850)

Familia Suguenziidae Verrill, 1884

Seguenzia hapala Woodring, 1928

Suborden Neritimorpha Golikov \& Starobogatov, 1975

Familia Neritidae Rafinesque, 1815

Neritina virginea (Linnaeus, 1758)

Familia Phenacolepadidae Thiele, 1929

Phenacolepas hamillei (Fischer, 1857)

Orden Mesogastropoda Thiele, 1925

Familia Littorinidae Gray, 1940

Littorina lineata d’Orbigny, 1841

Littorina (= Littoraria) flava King \& Broderip, 1832

Littorina ziczac (Gmelin, 1791)

Littorina (= Littoraria) angulifera (Lamarck, 1822)

Littorina lineolata d’Orbigny, 1840

Familia Pterotracheidae Rafinesque, 1814

Firoloida desmarestia Lesueur, 1817

Familia Hydrobiidae Troschel, 1857

(= Cochliopidae Tryon, 1866)

Heleobia (= Littoridina) australis (d'Orbigny, 1835)

Familia Rissoidae Gray, 1847

Benthonella tenella (Jeffreys, 1883)

Ceratia rustica (Watson, 1885)

Rissoina indiscreta Leal \& Moore, 1989

Rissoina (= Schwartziella) bryerea (Montagu, 1803)

Rissoina (= Schwartziella) catesbyana (d’Orbigny, 1842)
Alvania cruzi (Castellanos \& Fernández, 1974)

Familia Barleeidae Gray, 1857

Crassitoniela rubrooperculata (Castellanos, 1972)

Familia Assimineidae Adams \& Adams, 1856

Assiminea succinea (Pfeiffer, 1840)

Familia Caecidae Gray, 1850

Caecum brasilicum Folin, 1874

Caecum condylum Moore, 1969

Caecum eliezeri Absalão, 1997

Caecum pulchellum Stimpson, 1851

Caecum antillarum Carpenter, 1857

Caecum strigosum Folin, 1867

Caecum ryssotitum Folin, 1867

Meioceras (= Caecum) cornucopiae (Carpenter, 1858)

Familia Vitrinellidae Bush, 1897

Episcynia inornata (d’Orbigny, 1842)

Solariorbis mooreana (Vanatta, 1904)

Solariorbis shimeri (Clapp, 1914)

Solariorbis shumoi (Vanatta, 1913)

Teinostoma megastoma (C. B. Adams, 1850)

Familia Modulidae Fischer, 1884

Modulus modulus (Linnaeus, 1758)

Familia Cerithiidae Fleming, 1822

Cerithium atratum (Born, 1778)

Bittium varium (Pfeiffer, 1840)

Familia Diastomatidae Cossmann, 1893

Finella dubia (d’Orbigny, 1842)

Familia Potamididae Adams \& Adams, 1854

Batillaria minima (Gmelin, 1791)

Familia Fossaridae Troschel, 1861

Fossarus orbigny Fischer, 1854

Turritella hookeri Reeve, 1849

Familia Vermetidae Rafinesque, 1815

Dendropoma annulatus (Daudin, 1800)

Petaloconchus erectus (Dall, 1888)

Petaloconchus varians (d’Orbigny, 1841) 
Serpulorbis decussatus (Gmelin, 1791)

Familia Strombidae Rafinesque, 1815

Strombus pugilis Linnaeus, 1758

Familia Hipponicidae Troschell, 1861

Hipponyx grayanus Menke, 1853

Hipponyx subrufus (Lamarck, 1819)

Familia Calyptraeidae Lamarck, 1809

Crepidula aculeata (Gmelin, 1791)

Crepidula plana Say, 1822

Crepidula protea d’Orbigny, 1835

Crepidula pyguaia Simone, 2006

Calyptraea centralis (Conrad, 1841)

Familia Capulidae Fleming, 1822

Capulus incurvatus (Gmelin, 1791)

Familia Xenophoridae Troschel, 1852

Onustus (= Xenophora) longleyi Bartsch, 1931

Onustus (= Xenophora) caribaeus Petit, 1856

Familia Cypraeidae Rafinesque, 1815

Erosaria (= Cypraea) acicularis Gmelin, 1791

Cypraea (= Macrocypraea) zebra (Linnaeus, 1758)

Familia Ovulidae Fleming, 1822

Cyphoma signatum Pilsbry \& McGinty, 1939

Cyphoma intermedium (Sowerby, 1828)

Cyphoma gibsonsmithorum Petuch, 1987

Simnialena (= Simnia) uniplicata (Sowerby, 1848)

Familia Triviidae Troschel, 1863

Erato maugeriae (Gray, 1832)

Trivia maltbiana Schwengel \& McGinty, 1942

Familia Lamellariidae d’Orbigny, 1841

Lamellaria perspicua mopsicolor E. Marcus, 1956

Lamellaria patagonica E. A. Smith, 1881

Familia Atlantidae Wiegmamm \& Ruthe, 1832

Atlanta peroni Lesueur, 1817
Familia Naticidae Guilding, 1834

Natica limbata d'Orbigny, 1840

Natica pusilla Say, 1822

Natica isabelleana d'Orbigny, 1840

Naticarius (= Natica) canrena (Linnaeus, 1758)

Polinices hepaticus (Röding, 1798)

Polinices lacteus (Guilding, 1833)

Polinices uberinus (d’Orbigny, 1842)

Sinum maculatum (Say, 1831)

Sinum perspectivum (Say, 1831)

Familia Tonnidae Suter, 1913

Eudolium bairdii (Verrill \& Smith, 1881)

Tonna galea (Linnaeus, 1758)

Familia Cassidae Latreille, 1825

Cypraecassis testiculus (Linnaeus, 1758)

Phalium (=Semicassis) labiatum iheringi

Carcelles, 1953

Phalium (= Semicassis) granulatum (Born, 1778)

Eudolium crosseanum (Monterosato, 1869)

Semicassis granulatum peristephes (Pilsbry \& McGinty, 1939)

Familia Ranellidae Gray, 1854

Distorsio clathrata (Lamarck, 1816)

Ranella olearium barcellosi Mathews, Rios \& Coelho, 1973

Ranella australasia gemnifera (Euthyme, 1889)

Cabestana (= Cymatium) felipponei (Ihering, 1907)

Charonia lampas pustulata (Euthyme, 1889)

Cymatium femorale (Linnaeus, 1758)

Cymatium labiosum (Wood, 1828)

Cymatium nicobaricum (Roding, 1798)

Cymatium parthenopeum parthenopeum (von Salis, 1793)

Linatella caudata (Gmelin, 1791)

Linatella succinta (Linnaeus, 1771)

Fusitriton brasiliensis Cossignani \&

Cossignani, 2003

Fusitriton magellanicus (Röding, 1798)

Familia Bursidae Thiele, 1925

Bursa corrugata ponderosa (Reeve, 1844)

Bursa latitudo natalensis Coelho \& Mathews, 1970

Bursa ranelloides tenuisculpta (Dautzenberg \& Fischer, 1906) 
Familia Cerithiopsidae Adams \& Adams, 1853

Cerithiopsis bicolor (C. B. Adams, 1845)

Cerithiopsis flava (C.B. Adams, 1850)

Cerithiopsis gemmulosa (C. B. Adams, 1839)

Cerithiopsis greenii (C. B. Adams, 1839)

Cerithiopsis emersoni (C. B. Adams, 1838)

Seila adamsi (H. Lea, 1845)

Familia Triphoridae Gray, 1847

Triphora nigrocincta (C. B. Adams, 1839)

Triphora pulchella (C. B. Adams, 1850)

Familia Epitoniidae S.S. Berry, 1910

Epitonium albidum (d’Orbigny, 1842)

Epitonium angulatum (Say, 1830)

Epitonium georgettinum (Kiener, 1839)

Epitonium humpheysii (Kiener, 1838)

Epitonium krebsi (Morch, 1874)

Epitonium novangliae (Couthouy, 1838)

Epitonium nautlae (Morch, 1874)

Epitonium tenuistriatum (d’Orbigny, 1840)

Epitonium unifasciatum (Sowerby, 1844)

Epitonium candeanum (d’Orbigny, 1842)

Opalia crenata (Linnaeus, 1758)

Opalia hotessieriana (d’Orbigny, 1842)

Familia Janthinidae Leach, 1823

Janthina janthina (Linnaeus, 1758)

Janthina exigua Lamarck, 1816

Janthina pallida (Thompson, 1840)

Recluzia rollandiana Petit, 1853

Familia Eulimidae Risso, 1826

Eulima hemphilli (Dall, 1884)

Eulima mulata Rios \& Absalão, 1990

Balcis breviuscula (Dunker, 1875)

Melanella arcuata (C. B. Adams, 1850)

Melanella breviuscula (Dunker, 1875)

Melanella hebes (Watson, 1883)

Melanella sarissa (Watson, 1883)

Niso aeglees Bush, 1895

Familia Aclididae Sars, 1878

Aclis underwoodae (Bartsch, 1947)

Orden Neogastropoda Wenz, 1938

Familia Muricidae Rafinesque, 1815

Chicoreus (= Siratus) beauii (Fischer \& Bernardi, 1857)

Chicoreus (= Siratus) senegalensis (Gmelin, 1790)
Chicoreus (= Siratus) tenuivaricosus

(Dautzenberg, 1927)

Phyllonotus pomum ocularis (Reeve, 1845)

Muricopsis necocheana (Pilsbry, 1900)

Favartia cellulosa (Conrad, 1846)

Coronium (= Fulgurofusus) coronatum (Penna-

Neme \& Leme, 1978)

Coronium elegans Simone, 1996

Trachipollia turricula (von Maltzan, 1884)

Trachipollia (= Morula) nodulosa (C. B.

Adams, 1845)

Urosalpinx cala (Pilsbry, 1897)

Urosalpinx haneti (Petit, 1856)

Trophon pelseneeri E. A. Smith, 1915

Typhis cleryi (Petit, 1842)

Typhis riosi Bertsch \& D’Attilio, 1980

Familia Thaididae Jousseaume, 1888

Stramonita (= Thais) haemastoma (Linnaeus, 1767)

Thais mariae Morretes, 1954

Familia Coralliophilidae Chenu, 1859

Coralliophila aberrans (C. B. Adams, 1850)

Coralliophila caribaea Abbott, 1958

Latiaxis (= Babelomurex) dalli (Emerson \& D’Áttilio, 1963)

Latiaxis basilium Neme \& Leme, 1978

Familia Buccinidae Rafinesque, 1815

Cantharus auritula (Link, 1807)

Metula anfractura Matthews \& Rios, 1969 (= Metula agassizi Clench \& Aguayo, 1941)

Metula gigliottii Coltro, 2005

Pisania auritula (Link, 1807)

Pisania pusio (Linnaeus, 1758)

Gemophos auritulus (Link, 1807)

Familia Columbellidae Swainson, 1840

Aesopus metcalfei (Reeve, 1858)

Amphissa cancellata (Castellanos, 1982)

Amphissa acuminata (E. A. Smith, 1915)

Anachis (= Costoanachis) catenata (Sowerby, 1844)

Anachis fenneli Radwin, 1968

Anachis lyrata (Sowerby, 1832)

Anachis pulchella (Blainville, 1829)

Anachis sertulariarum d’Orbigny, 1841

Anachis (= Costoanachis) sparsa (Reeve, 1859)

Anachis isabellei (d'Orbigny, 1841)

Anachis (= Parvanachis) obesa (C. B. Adams, 1845)

Anachis veleda (Duclos, 1846)

Mazatlania fulgurata (Phillippi, 1846) 
Mitrella argus (d'Orbigny, 1842)

Mitrella (= Astyris) lunata (Say, 1826)

Mitrella pusilla (Sowerby, 1844)

Suturoglypta hotessieriana (d’Orbigny, 1842)

Familia Nassariidae Iredale, 1916

Nassarius albus (Say, 1826)

Nassarius vibex (Say, 1822)

Nassarius scissuratus (Dall, 1889)

Dorsanum moniliferum (Valenciennes, 1834)

Buccinanops duartei Klappenbach, 1961

Buccinanops gradatum (Deshayes, 1844)

Buccinanops lamarckii (Kiener, 1834)

Buccinanops uruguayensis (Pilsbry, 1897)

Buccinanops deformis (King \& Broderip, 1832)

Familia Melongenidae Grill, 1867

Pugilina morio (Linnaeus, 1758)

Familia Fasciolariidae Gray, 1853

Fusinus frenguelli (Carcelles, 1953)

Fusinus marmoratus (Philippi, 1846)

Leucozonia nassa (Gmelin, 1791)

Familia Volutidae Rafinesque, 1815

Zidona dufresnei (Donovan, 1823)

Zidona dufresneyi disctincta (Lahille, 1895)

Adelomelon ancilla (Lightfoot, 1786)

Adelomelon beckii (Broderip, 1836)

Adelomelon (= Pachycymbiola) brasiliana (Lamarck, 1811)

Adelomelon riosi Clench \& Turner, 1964

Odontocymbiola americana (Reeve, 1856)

Odontocymbiola (= Minicymbiola) corderoi

(Carcelles, 1953)

Odontocymbiola magellanica (Gmelin, 1791)

Odontocymbiola simulatrix Leal \& Bouchet, 1989

Odontocymbiola macaensis Calvo \& Coltro, 1997

Nanomelon viperinus Leal \& Bouchet, 1989

Familia Olividae Latreille, 1825

Oliva circinata Marrat, $1870 \quad$ (= Oliva

reticularis Lamarck, 1811)

Oliva fulgurator (Röding, 1798)

Olivancillaria carcellesi Klappenbach, 1965

Olivancillaria contortuplicata (Reeve, 1850)

Olivancillaria deshayesiana (Duclos, 1857)

Olivancillaria uretai Klappenbach, 1965

Olivancillaria urceus (Roding, 1798)

Olivancillaria vesica vesica (Gmelin, 1791)
Olivancillaria vesica auricularia (Lamarck, 1810)

Ancilla dimidiata (Sowerby, 1850)

Olivella nivea (Gmelin, 1791)

Olivella petiolita (Duclos, 1835)

Olivella defiorei Klappenbach, 1964

Olivella minuta (Link, 1807)

Olivella orejasmirandai Klappenbach, 1986

Olivella puelcha (Duclos, 1840)

Olivella formicacorsii Klappenbach, 1962

Agaronia travassosi Morretes, 1938

Amalda josecarlosi Pastorino, 2003

Familia Marginellidae Lamarck, 1799

Prunum (= Marginella) martini (Petit, 1853)

Prunum (= Marginella) rubens (Martens, 1881)

Volvarina pontesi Rios \& Leal, 1993

Familia Mitridae Swainson, 1831

Mitra anfractura Mattews \& Rios, 1970

Mitra antillensis Dall, 1889

Mitra larranagai (Carcelles, 1947)

Familia Costellariidae MacDonald, 1860

Thala crassa Simone, 1995

Suborden Toxoglossa Troschel, 1848

Familia Conidae Rafinesque, 1815

Conus carcellesi Martins, 1945

Conus clerii Reeve, 1844

Conus clenchi Martins, 1943

Conus lemniscatus Reeve, 1849

Conus villepinii Fischer \& Bernardi, 1867

Conus xanthocinctus Petuch, 1986

Conus mazei Deshayes, 1874

Conus tostesi Petuch, 1979

Familia Turridae Swainson, 1840

Cerodrillia perryae Bartsch \& Rehder, 1939

Drilliola (= Microdrillia) comatotropis (Dall, 1881)

Drilliola loprestiana (Calcara, 1841)

Splendrillia lissotropis (Dall, 1881)

Polystira formosissima (E. A. Smith, 1915)

Carinodrillia braziliensis (E. A. Smith, 1915)

Fusiturricula taurina (Olsson, 1922)

Gemmula periscelida (Dall, 1875)

Gemmula mystica Simone, 2005

Compsodrillia tristicha (Dall, 1889)

Pilsbryspira albomaculata (d’Orbigny, 1842)

Cochlespira elegans (Dall, 1881)

Cochlespira elongata Simone, 1999 
Brachytoma rioensis (E. A. Smith, 1915)

Stenodrillia (= Knefastia) horrenda (Watson, 1885)

Glyphostoma epicasta (Bartsch, 1934)

Mangelia fusca C. B. Adams, 1850

Mangelia quadrata (Reeve, 1845)

Mangelia rugirima (Dall, 1889)

Cryoturris adamsi (E. A. Smith, 1884)

Kurtziella rhysa (Watson, 1881)

Kurtziella dorvillae (Reeve, 1845)

Granoturris (= Kurtziella) padolina Fargo, 1953

Ithycythara hyperlepta Haas, 1953

Ithycythara lanceolata (C. B. Adams, 1850)

Pyrgocythara albovittata (C. B. Adams, 1845)

Pyrgocythara caribaea (d’Orbigny, 1842)

Pyrgocythara guarani (d’Orbigny, 1841)

Tenaturris fulgens (E. A. Smith, 1888)

Pleurotomella aguayoi (Carcelles, 1953)

Pleurotomella circumvoluta (Watson, 1881)

Veprecula morra (Dall, 1881)

Fenimorea sp.

Familia Terebridae Mörch, 1852

Terebra brasiliensis (E. A. Smith, 1873)

Terebra doello-juradoi Carcelles, 1953

Terebra gemmulata Kiener, 1839

Terebra riosi Bratcher \& Cernohorsky, 1985

Terebra spirosulcata Simone \& Costa, 1999

Terebra taurina (Lightfoot, 1786)

Hastula cinerea (Born, 1778)

Hastula hastata (Gmelin, 1791)

Orden Heterostropha Fisher, 1885

Familia Architectonicidae Gray, 1840

Architectonica nobilis Roding, 1798

Heliacus bisulcatus (d’Orbigny, 1845)

Philippia uruguaya (Carcelles, 1953)

Familia Pyramidellidae Gray, 1840

Odostomia canaliculata C. B. Adams, 1850

Odostomia laevigata (d’Orbigny, 1842)

Odostomia muelleri Clessin, 1900

Odostomia bisuturalis ( Say, 1822 )

Odostomia seminuda (C. B. Adams, 1837)

Fargoa (= Odostomia) bushiana Bartsch, 1909

Chrysallida (=Odostomia) jadisi Olsson \&

McGinty, 1958

Peristichia agria Dall, 1889

Turbonilla uruguayensis Pilsbry, 1897

Turbonilla pusilla (C. B. Adams, 1850)

Turbonilla americana (d’Orbigny, 1840)

Turbonilla atypha Bush, 1899

Turbonilla brasiliensis Clessin, 1900

Turbonilla dispar Pilsbry, 1897

Turbonilla fasciata (d’Orbigny, 1840)
Turbonilla farrouphila Pimenta \& Absalão, 2004

Turbonilla interrupta (Totten, 1835)

Turbonilla haycocki Dall \& Bartsch, 1911

Turbonilla kaapor Pimenta \& Absalão, 2004

Turbonilla multicosta (C. B. Adams, 1850)

Turbonilla abrupta Bush, 1899

Turbonilla goytacazi Pimenta \& Absalão, 2004

Turbonilla turris (d’Orbigny, 1840)

Turbonilla rushi Bush, 1899

Familia Amathnidae Ponder, 1988

Iselica anomala (C. B. Adams, 1850)

Subclasse Opisthobranchia Milne Edwards, 1848

Orden Cephalaspidea P. Fischer, 1883

Familia Acteonidae d'Orbigny, 1842

Acteon pelecais Marcus, 1981

Mysouffa cumingii (A. Adams, 1854)

Familia Cylichnidae H. \& A. Adams, 1854

Cylichna crispula Watson, 1883

Cylichna verrillii Dall, 1889

Acteocina bullata (Kiener, 1834)

Acteocina bidentata (d’Orbigny, 1841)

Acteocina candei (d’Orbigny, 1842)

Acteocina inconspicua Olsson \& McGinty, 1958

Familia Retusidae Thiele, 1926

Pyrunculus caelatus (Bush, 1885)

Volvulella persimilis (Morch, 1875)

Familia Hamineidae Pilsbry, 1895

Haminoea elegans (Gray, 1825)

Familia Bullidae Rafinesque, 1815

Bulla striata Bruguìere, 1792

Orden Anaspidea Fischer, 1883

Familia Aplysiidae Lamarck, 1809

Aplysia juliana Quoy \& Gaimard, 1823

Aplysia brasiliana Rang, 1828

Aplysia dactylomela Rang, 1828

Bursatella leachi Blainville, 1817

Orden Notaspidea Fischer, 1883

Familia Umbraculidae Dall, 1889

Umbraculum sp. 
Familia Pleurobranchidae Férussac, 1822

Pleurobranchaea inconspicua Bergh, 1897 Pleurobranchus areolatus Morch, 1836 Pleurobranchus testudinarius Cantraine, 1835

Orden Thecosomata Blainville, 1824

Familia Cavoliniidae Fischer, 1883

Cavolinia gibbosa (d’Orbigny, 1836)

Cavolinia inflexa imitans (Pfeffer, 1879)

Cavolinia tridentata Niebuhr, 1775

Cavolinia uncinata (Rang, 1813)

Diacria trispinosa (Blainville, 1821)

Cuvierina spoeli Rampal, 2002

Clio pyramidata lanceolata (Lesueur, 1813)

Creseis acicula (Rang, 1802)

Orden Nudibranchia Blainville, 1814

Familia Polyceridae Alder \& Hancock, 1845

Polycera aurisula Marcus, 1957

Roboastra ernsti Pola, Padula, Gosliner \& Cervera, 2014

Tambja brasiliensis Pola, Padula, Gosliner \& Cervera, 2014

Tambja stegosauriformis Pola, Cervera \& Gosliner, 2005

Familia Chromodorididae Bergh, 1879

Chromodoris paulomarcioi Dominguez, Garcia \& Troncoso, 2006

Hypselodoris (= Felimare) lagensis (Troncoso, Garcia \& Urgorri, 1998)

Felimida (= Hypselodoris) marci (Marcus, 1971)

Tyrinna (= Okenia) evelinae (Marcus, 1958)

Familia Dorididae Rafinesque, 1815

Doris verrucosa Cuvier, 1804

Etidoris ladislavii Ihering, 1886

Familia Dendrodorididae Pruvot-Fol, 1935

Dendrodoris krebsi (Morch, 1863)

Familia Tritoniidae Menke, 1828

Marionia cucullata (Gould, 1852)

Tritonia eriosi Marcus, 1983

Familia Arminidae Pruvot-Fol, 1927

Armina muelleri (Ihering, 1886)
Familia Flabellinidae Bergh in Carus, 1889

Flabellina engeli lucianae Da Costa, Cunha, Simone \& Schrodl, 2007

Flabellina marcusorum Gosliner \& Kuzirian, 1990

Familia Tergipedidae Bergh, 1889

Cuthona iris Edmunds \& Just, 1983

Familia Glaucidae Menke, 1828

Glaucus atlanticus Foster, 1777

Phidiana lynceus Bergh, 1867

Dondice occidentalis Engel, 1925

Familia Aeolidiidae d’Orbigny, 1834

Anteaeolidiella (= Aeolidiella) indica (Bergh, 1888)

Berghia rissodominguezi Muniain \& Ortea, 1999

Spurilla neapolitana (Delle Chiaje, 1923)

Familia Onchidiidae Rafinesque, 1815

Onchidella indolens (Gould, 1852)

Subclasse Pulmonata Cuvier, 1817

Orden Archaeopulmonata Morton, 1955

Familia Ellobiidae H. \& A. Adams, 1865

Melampus coffea (Linnaeus, 1758)

Pedipes mirabilis (Mohlfeld, 1816)

Orden Basommatophora A. Schmidt, 1855

Familia Siphonariidae Gray, 1840

Siphonaria hispida E. A . Smith, 1890

Siphonaria lessoni (Blainville, 1824)

Suborden Eupulmonata Haszprunar \& Huber, 1990

Familia Trimusculidae J. Q. Burch, 1945 (1840)

Trimusculus (= Gadinia) goesi (Hubendick, 1946)

Classe Scaphopoda Bronn, 1862

Orden Dentaliida da Costa, 1776

Familia Dentaliidae Gray, 1834

Dentalium (Dentalium) americanum Chenu, 1843

Dentalium (Dentalium) laqueatum Verrill, 1885 
Dentalium (Heteroschismoides) callithrix Dall, 1889

Dentalium (Antalis) ceratum Dall, 1881

Dentalium (Antalis) disparile d’Orbigny, 1842

Dentalium (Antalis) infractum Odhner, 1931

Dentalium (Fissidentalium) carduum (= Dentalium (Fissidentalium) floridense (Dall, 1889))

Graptacme perlongum (Dall, 1881)

Graptacme semistriolatum (Guilding, 1834)

Familia Gadilidae Stoliczka, 1868

Polyschides tetraschistus (Watson, 1879)

Classe Bivalvia Linnaeus, 1758

Subclasse Protobranchia Woodward, 1892

Ordem Nuculoida Dall, 1889

Familia Nuculidae Gray, 1824

Nucula semiornata d’Orbigny, 1846

Nucula puelcha d’Orbigny, 1846

Familia Nuculanidae Adams \& Adams, 1858

Nuculana larranagai Klappenbach \&

Scarabino, 1968

Adrana electa (A. Adams, 1846)

Adrana patagonica (d’Orbigny, 1846)

Familia Neilonellidae Schileyko, 1989

Neilonella acinula Dall, 1889

Subclasse Pteriomorpha Beurlen, 1944 Orden Arcoida Stoliczka, 1871

Familia Arcidae Lamarck, 1809

Arca imbricata Bruguiere, 1789

Barbatia cancellaria (Lamarck, 1819)

Barbatia dominguensis (Lamarck, 1819)

Barbatia candida (Helbling, 1779)

Anadara brasiliana (Lamarck, 1819)

Anadara chemnitzi (Philippi, 1851)

Anadara notabilis (Roding, 1798)

Anadara (Lunarca) ovalis (Bruguìere, 1789)

Familia Noetiidae Stewart, 1930

Noetia bisulcata (Lamarck, 1819)

Arcopsis adamsi (Dall, 1886)

Familia Limopsidae Dall, 1895

Limopsis janeiroensis E. A. Smith, 1915
Familia Glycymerididae Newton, 1922

Glycymeris longior (Sowerby, 1833)

Glycymeris undata (Linnaeus, 1758)

Glycymeris pectinata (Gmelin, 1791)

Orden Mytiloida Férussac, 1822

Familia Mytilidae Rafinesque, 1815

Brachidontes exustus (Linnaeus, 1758)

Brachidontes solisianus (d'Orbigny, 1846)

Crenella decussatta (Montagu, 1808)

= Crenella divaricata (d'Orbigny, 1853)

Mytella charruana (d’Orbigny, 1842)

Mytella guyanensis (Lamarck, 1819)

Perna perna (Linnaeus, 1758)

Modiolus carvalhoi Klappenbach, 1966

Lioberus castaneus (Say, 1822)

Gregariella coralliophila (Gmelin, 1791)

Musculus lateralis (Say, 1822)

Musculus viator (d’Orbigny, 1846)

Lithophaga bisulcata (d'Orbigny, 1842)

Lithophaga patagonica (d’Orbigny, 1847)

Myoforceps aristatus (Dillwyn, 1817)

Familia Pteriidae Gray, 1847

Pteria hirundo (Linnaeus, 1758)

Pinctada imbricata Roding, 1798

Familia Isognomonidae Woodring, 1925

Isognomon bicolor (C. B. Adams, 1845)

Familia Pinnidae Leach, 1819

Pinna carnea Gmelin, 1791

Atrina seminuda (d’Orbigny, 1846)

Familia Limidae Rafinesque, 1815

Lima scabra (Born, 1778)

Limaria inflata Lamarck, 1819

Limaria pellucida (C. B. Adams, 1846)

Orden Ostreoida Waller, 1978

Familia Ostreidae Rafinesque, 1815

Ostrea cristata Born, 1778

Ostreola (= Ostrea) equestris (Say, 1834)

Ostrea puelchana d'Orbigny, 1841

Crassostrea gigas (Thunberg, 1795)

Crassostrea rhizophorae (Guilding, 1828)

Crassostrea brasiliana Lamarck, 1819

Crassostrea mangle Amaral \& Simone, 2014 
Crassostrea virginica (Gmelin, 1791)

Dendostrea (= Lopha) frons (Linnaeus, 1758)

Familia Plicatulidae Watson, 1930

Plicatula gibbosa Lamarck, 1801

Familia Pectinidae Rafinesque, 1815

Chlamys felipponei (Dall, 1922)

Chlamys muscosus (Wood, 1828)

Chlamys sentis (Reeve, 1853)

Chlamys tehuelchus (d’Orbigny, 1846)

Argopecten (= Chlamys) gibbus (Linnaeus, 1758)

Argopecten noronhensis (E. A. Smith, 1885)

Leptopecten bavary (Dautzenberg, 1900)

Nodipecten (= Lyropecten) nodosus (Linnaeus, 1758)

Euvola (= Pecten) ziczac (Linnaeus, 1758)

Cyclopecten nanus Verrill \& Bush, 1897

Familia Spondylidae Gray, 1826

Spondylus ictericus Reeve, 1856

Familia Anomiidae Rafinesque, 1815

Anomia ephippium Linnaeus, 1758

Pododesmus rudis (Broderip, 1834)

Orden Veneroida Adams \& Adams, 1856

Familia Lucinidae Fleming, 1828

Lucina pectinata (Gmelin, 1791)

Lucina rehderi Britton, 1972

Codakia costata (d'Orbigny, 1842)

Codakia orbicularis (Linnaeus, 1758)

Codakia (Ctena) pectinella C. B. Adams, 1852

Ctena (= Codakia) orbiculata (Montagu, 1808)

Linga amiantus (Dall, 1901)

Myrtea lens (Verill \& Smith, 1880)

Lucina tixierae (Klein, 1969)

Divaricella quadrisulcata (d’Orbigny, 1842)

Familia Thyasiridae Dall, 1901

Thyasira trisinuata d’Orbigny, 1842

Familia Ungulinidae Adams \& Adams, 1857

Diplodonta danieli Klein, 1967

Diplodonta patagonica (d’Orbigny, 1842)

Diplodonta punctata (Say, 1822)

Felaniela candeana (d’Orbigny, 1842)

Felaniela vilardeboana (d’Orbigny, 1846)
Phlyctiderma semiaspera (Philippi, 1836)

Familia Sportellidae Dall, 1899

Basterotia elliptica (Recluz, 1850)

Basterotia quadrata (Hinds, 1843)

Familia Carditidae Fleming, 1820

Carditamera floridana Conrad, 1838

Carditamera plata (Ihering, 1907)

Familia Condylocardiidae Bernard, 1897

Carditopsis smithii (Dall, 1896)

Familia Chamidae Lamarck, 1809

Chama congregata Conrad, 1833

Chama macerophylla (Gmelin, 1791)

Chama sarda Reeve, 1847

Pseudochama radians (Lamarck, 1819)

Arcinella brasiliana (Nicol, 1953)

Familia Crassatellidae Férussac, 1822

Crassatella brasiliensis (Dall, 1903)

Crassatella riograndensis Vokes, 1973

Crassinella lunulata (Conrad, 1834)

Crassinella marplatensis Castellanos, 1970

Crassinella martinicensis (d’Orbigny, 1842)

Familia Cardiidae Lamarck, 1809

Trachycardium (= Dallocardia) manueli (Prado, 1993)

Trachycardium muricatum (Linnaeus, 1758)

Papyridea soleniformis (Bruguière, 1789)

Nemocardium (= Microcardium) tinctum (Dall, 1881)

Laevicardium brasilianum (Lamarck, 1819)

Laevicardium laevigatum (Linnaeus, 1758)

Familia Mactridae Lamarck, 1809

Mactra isabelleana d’Orbigny, 1846

Mactra marplatensis Doello-Jurado, 1918

Mactra patagonica d'Orbigny, 1846

Mactra fragilis Gmelin, 1791

Mactra iheringi Dall, 1897

Mactra petiti d'Orbigny, 1846

Mactra janeiroensis E. A. Smith, 1915

Mactrellona alata (Spengler, 1802)

Mulinia cleryana (d’Orbigny, 1846)

Anatina anatina (Spengler, 1802)

Raeta plicatella (Lamarck, 1818) 
Familia Mesodesmatidae Gray, 1839

Mesodesma mactroides Deshayes, 1854

Familia Solenidae Lamarck, 1809

Solen tehuelchus d'Orbigny, 1843

Solen obliquus Spengler, 1794

Familia Tellinidae Blainville, 1814

Tellina radiata Linnaeus, 1758 (= Tellina brasiliana Spengler, 1798)

Tellina diantha Boss, 1964

Tellina exerythra Boss, 1964

Tellina versicolor De Kay, 1843

Tellina alternata Say, 1822

Tellina angulosa Gmelin, 1791

Tellina lineata Turton, 1819

Tellina nitens C. B. Adams, 1845

Tellina punicea Born, 1778

Tellina aequistriata Say, 1824

Tellina alerta Boss, 1964

Tellina martinicensis d’Orbigny, 1853

Tellina sandix Boss, 1968

Tellina listeri Roding, 1798

Eurytellina angrensis Marques \& Simone, 2014

Strigilla carnaria (Linnaeus, 1758)

Strigilla pisiformis (Linnaeus, 1758)

Macoma cleryana (d'Orbigny, 1846)

Macoma tenta (Say, 1834)

Macoma constricta (Bruguière, 1792)

Macoma brevifrons (Say, 1834)

Macoma uruguayensis (E. A. Smith, 1885)

Temnoconcha brasiliana Dall, 1921

Familia Semelidae Stoliczka, 1870

Semele casali Doello-Jurado, 1949

Semele proficua (Pulteney, 1799)

Semele purpurascens (Gmelin, 1791)

Semele nuculoides (Conrad, 1841)

Semele modesta (Reeve, 1853)

Abra aequalis (Say, 1822)

Abra lioica (Dall, 1881)

Ervilia concentrica (Holmes, 1860)

Familia Psammobiidae Fleming, 1828

Sanguinolaria sanguinolenta (Gmelin, 1791)

Sanguinolaria cruenta (Lightfoot, 1786)

Solecurtus cumingianus (Dunker, 1861)

Solecurtus sanctaemarthae Orbigny, 1842

Gari sp.

Tagelus plebeius (Lightfoot, 1786)
Tagelus divisus (Spengler, 1794)

Familia Donacidae Fleming, 1828

Donax gemmula Morrison, 1971

Donax hanleyanus Philippi, 1842

Iphigenia brasiliana (Lamarck, 1818)

Familia Gaimardiidae Hedley, 1916

Gaimardia trapezina (Lamarck, 1819)

Familia Veneridae Rafinesque, 1815

Ventricolaria rigida (Dillwyn, 1817)

Gouldia cerina (C. B. Adams, 1845)

Chione cancellata (Linnaeus, 1767)

Chione pubera (Bory Saint-Vicent, 1827)

Chione paphia (Linnaeus, 1767)

Anomalocardia brasiliana (Gmelin, 1791)

Protothaca antiqua (King \& Broderip, 1835)

Protothaca pectorina (Lamarck, 1818)

Tivela fulminata (Valenciennes, 1827)

Tivela mactroides (Born, 1778)

Tivela ventricosa (Gray, 1838)

Tivela isabelleana (d’Orbigny, 1846)

Tivela foresti Fischer-Piette \& Testud, 1967

Transenella stimpsoni Dall, 1902

Pitar fulminatus (Menke, 1828)

Pitar palmeri Fischer-Piette \& Testud, 1967

Pitar rostratus (Koch, 1844)

Pitar circinatus (Born, 1778)

Amiantis purpuratus (Lamarck, 1818)

Callista (= Macrocallista) maculata (Linnaeus, 1758)

Transenpitar americana (Doello-Jurado, 1951)

Dosinia concentrica (Born, 1778)

Cyclinella tenuis (Récluz, 1852)

Familia Petricolidae Deshayes, 1831

Petricola lapicida (Gmelin, 1791)

Petricola stellae Narchi, 1975

Petricola typica (Jonas, 1844)

Orden Myoidea Stoliczka, 1870

Familia Myidae Lamarck, 1809

Sphenia antillensis Dall \& Simpson, 1901

Familia Corbulidae Lamarck, 1818

Corbula caribaea Orbigny, 1842

Corbula lyoni Pilsbry, 1897

Corbula cubaniana d’Orbigny, 1853 
Familia Erodonidae Winckworth, 1932

Erodona mactroides Bosc, 1802

Familia Gastrochaenidae Gray, 1840

Gastrochaena hians (Gmelin, 1791)

Familia Hiatellidae Gray, 1824

Hiatella arctica (Linnaeus, 1767)

Panopea abbreviata Valenciennes, 1839

Familia Pholadidae Lamarck, 1809

Pholas campechiensis Gmelin, 1791

Barnea truncata (Say, 1822)

Cyrtopleura costata (Linnaeus, 1758)

Cyrthopleura lanceolata (d'Orbigny, 1846)

Martesia striata (Linnaeus, 1758)

Martesia fragilis Verrill \& Bush, 1890

Martesia cuneiformis (Say, 1822)

Familia Teredinidae Rafinesque, 1815

Teredo navalis Linnaeus, 1758

Teredo bartschi Clapp, 1923

Teredo fulleri Clapp, 1924

Lyrodus floridanus (Bartsch, 1922)

Lyrodus pedicellatus (Quatrefages, 1849)

Psiloteredo healdi (Bartsch, 1922)

Neoteredo reynei (Bartsch, 1920)

Spathoteredo spatha (Jeffreys, 1860)

Nausitora fusticula (Jeffreys, 1860)

Bankia carinata (Gray, 1827)

Bankia gouldi Bartsch, 1908

Bankia destructa Clench \& Turner, 1946

Bankia cieba Clench \& Turner, 1946

Bankia bagidaensis (Roch, 1931)

Bankia rochi (Moll, 1931)

Bankia setacea (Tryon, 1863)

Bankia martensi (Stempell, 1899)

Familia Thraciidae E. A. Smith, 1885

Thracia similis Couthony, 1839

Asthenothaerus rushii (Pilsbry, 1897)

Familia Periplomatidae Dall, 1895

Periploma ovata d’Orbigny, 1846

Familia Pandoridae Rafinesque, 1815

Pandora arenosa Conrad, 1834

Pandora bushiana Dall, 1886
Pandora braziliensis Sowerby, 1874

Familia Cuspidariidae Dall, 1886

Cardiomya ornatissima (d’Orbigny, 1842)

Cardiomya perrostrata (Dall, 1881)

Familia Verticordiidae Stoliczka, 1871

Spinosipella agnes Simone \& Cunha, 2008

Classe Cephalopoda Bronn, 1798

Subclasse Coleoidea Bather, 1888

Orden Spirulida Stolley, 1919

Familia Spirulidae Owen, 1836

Spirula spirula Lamarck, 1801

Orden Sepiolida Fioroni, 1981

Familia Sepiolidae Leach, 1817

Semirrosia tenera (Verril, 1880)

Orden Teuthida Naef, 1916

Familia Loliginidae Lesueur, 1821

Loligo sanpaulensis Brakoniecki, 1984

(= Loligo brasiliensis Blainville, 1823)

Doryteuthis (= Loligo) plei (Blainville, 1823)

Lollinguncula brevis (Blainville, 1823)

Familia Ancistrocheiridae Pfeffer, 1912

Ancistrocheirus lesueuri (d’Orbigny, 1942)

Familia Architeuthidae Pfeffer, 1900

Architeuthis sp.

Familia Enoploteuthidae Pfeffer, 1900

Abralia veranyi (Rüppel, 1844)

Abralia redfieldi Voss, 1955

Familia Histioteuthidae Verrill, 1881

Histioteuthis meleagroteuthis (Chun, 1910)

Familia Lycoteuthidae Pfeffer, 1908

Lycoteuthis lorigera (Steenstrup, 1875)

Familia Ommastrephidae Steenstrup, 1857

Illex argentinus Castellanos, 1960

Ornithoteuthis antillarum Adam, 1957 
Ommastrephes bartramii (Lesueur, 1821)

Familia Onychoteuthidae Gray, 1849

Moroteuthis ingens (Smith, 1881)

Familia Thysanoteuthidae Keferstein, 1866

Thysanoteuthis rhombus Troschel, 1857

Orden Octopoda Leach, 1818

Familia Argonautidae Naef, 1912

Argonauta nodosa Lightfoot, 1786

Familia Octopodidae d’Orbigny, 1845

Octopus vulgaris Cuvier, 1797

Octopus tehuelchus d’Orbigny, 1834

Eledone massyae Voss, 1964

Eledone gaucha Haimovici, 1988

Vosseledone charrua Palacio, 1978

Scaeurgus unicirrhus (d’Orbigny, 1840)

\section{Consideraciones finales}

Resulta importante destacar que Rios (2009: 415) cita la espécie Bursatella leachi Blainville, 1817 en la Familia Notarchidae Eales, 1925, en vez de la Familia Aplysiidae Lamarck, 1809.

Apenas dos de las espécies relacionadas para el Estado son contempladas por categorias oficiales de conservación, siendo todavia consideradas como “amenazadas de extinción” (AgudoPadrón, 2015b): Gastropoda Olividae Olivancillaria contortuplicata (Reeve, 1850) y Bivalvia Pectinidae Euvola (= Pecten) ziczac (Linnaeus, 1758).

Otras cuatro constituyen reconocidas formas exóticas invasoras (Agudo-Padrón, 2011a): Bivalvia Ostreidae Crassostrea gigas (Thumberg, 1795) y Crassostrea virginica (Gmelin, 1791), Isognomonidae Isognomon bicolor (C. B. Adams, 1845), y Mytilidae Lithophaga (Myoforceps) aristatus (Dillwyn, 1817).

Finalmente, por lo menos las siguientes 10 encuentranse directamente envolvidas en actividades antrópicas de maricultura, pesca y extractivismo (AgudoPadrón y Bleicker, 2009b): Bivalvia Ostreidae Crassostrea gigas (Thunberg, 1795), Crassostrea virginica (Gmelin, 1791) y Crassostrea rhizophorae (Guilding,
1828), Mytilidae Perna perna (Linnaeus, 1758), Pectinidae Nodipecten nodosus (Linnaeus, 1758), Pteriidae Pteria hirundo (Linnaeus, 1758), Veneridae Anomalocardia brasiliana (Gmelin, 1791), asi como los Cephalopoda Loliginidae Doryteuthis (=Loligo) plei (Blainville, 1823), Ommastrephidae Illex argentinus Castellanos, 1960, y Octopodidae Octopus vulgaris Cuvier, 1797.

Confrontando los resultados cadastrales aquí presentados con las previas informaciones regionales disponibles para espécies de ambientes no marinos/ continentales - aquellas ocurrentes en ecosistemas terrestres y límnicos/ de agua dulce (Agudo-Padrón, 2014a, 2015), tenemos que el Estado de Santa Catarina finalmente cuenta hoy con amplio inventário/ ordenamiento de su riqueza faunística de moluscos, incluyendo un total verificado de 895 taxones (Tabla 1), siendo 671 formas marinas y 224 continentales 154 terrestres \& 70 límnicas/ de agua dulce, pasando de esta manera a constituir uno de los pocos Estados brasileños que, actualmente, cuentan con levantamientos básicos malacofaunisticos formalmente conocidos, revisados e permanentemente actualizados en sus territorios, conocimiento este fundamental para el desarrollo de necesarias y urgentes estrategias orientadas al alcance de su efectiva conservación (Agudo-Padrón, 2015a: 3, 6; 2015b).

\section{Declaración de conflicto de intereses}

El autor declara que no tiene ningún conflicto de intereses.

\section{Referências}

Agudo-Padrón, A. I. Exotic molluscs in Santa Catarina's State, Southern Brazil Region (Mollusca, Gastropoda et Bivalvia): check list and regional spatial distribution knowledge. Biodiversity Journal, v. 2, p. 53-58, 2011a. Disponible en: <http://www.biodiversityjournal. com/pdf/2_53-58.pdf $>$. Consultado el 15 abr. 2015.

Agudo-Padrón, A. I. Evaluative summary of the Santa Catarina's State mollusk fauna, Central Southern Brazil, after 15 years of research. FMCS Newsletter Ellipsaria, v. 13, p. 37-46, (2011b). Disponible en: 
<http://molluskconservation.org/EVENTS/

ELLIPSARIA/EllipsariaDec2011.pdf>.

Consultado el 15 abr. 2015.

Agudo-Padrón, A. I. Mollusc fauna in the Atlantic slope region of the Southern Cone of South America: a preliminary biogeographical interpretation. International Journal of Aquaculture, v. 2, p. 15-20, 2012. Disponible en: <http://biopublisher.ca/html-442-33-ija> . Consultado el 15 abr. 2015.

Agudo-Padrón, A. I. Inventario sistemático de los moluscos continentales ocurrentes en el Estado de Santa Catarina, Brasil. Bioma, v. 2, p. 6-23, 2014a. Disponible en: $<$ https://www.dropbox.com/s/cbfm6z3jsu1nghk/ Bioma Julio 2014.pdf $>$. Consultado el 15 abr. 2015.

Agudo-Padrón, A. I. Thirteen new records to inventory of marine mollusk species from Santa Catarina State, Central Southern Brazil. Brazilian Journal of Biological Sciences, v. 1, n. 2, p. 35-37, 2014b. Disponible en: $<$ http://revista.rebibio.net/v1n2/3543-4376-0106.html>. Consultado el 15 abr. 2015.

Agudo-Padrón, A. I. Molluscs of Santa Catarina State/SC, Central Southern Brazil: increments to species inventory, new geographical records and additional informations. International Journal of Aquaculture, v. 5, p. 1-8, 2015a. Disponible en: <http://www.sophiapublisher.com/ epublication-05/IJA/Vol.5/2/>. Consultado el 15 abr. 2015.

Agudo-Padrón, A. I. Balance of the brazilian molluscs "officially recognized" as threatened of extinction, with special emphasis in species occurring in the southern region. Brazilian Journal of Biological Sciences, v. 2, n. 3, p. 173-175, 2015b. Disponible en: $<$ http://revista.rebibio.net/v2n3/

v02n03a18a.html>. Consultado el 30 jun. 2015.

Agudo-Padrón, A. I.; Bleicker, M. S. Malacofauna marinha catarinense: estado atual do conhecimento dos moluscos marinhos. V: acréscimos ao cadastro de espécies. Informativo SBMa, v. 39, p. 8-10, 2008. Disponible en:

$<$ http://sbmalacologia.com.br/wp-content/ uploads/2011/09/Info-SBMa-39-164-30-062008.pdf>. Consultado el 15 abr. 2015.

Agudo-Padrón, A. I.; Bleicker, M. S. Malacofauna marinha catarinense. VI. Novos acréscimos ao cadastro de espécies. Informativo SBMa, v. 40, 8, 2009a. Disponible en: $\quad<$ http://sbmalacologia.com.br/wpcontent/uploads/2010/09/Info-40-1681.pdf>. Consultado el 15 abr. 2015.

Agudo-Padrón, A. I.; Bleicker, M. S. Malacofauna marinha catarinense. VII.
Aspectos gerais do seu conhecimento. Informativo SBMa, v. 40, p. 4-11, $2009 \mathrm{~b}$. Disponible en: <http://sbmalacologia.com.br/ wp-content/uploads/2011/08/Informativo_ 40_169Set2009.pdf>. Consultado el 15 abr. 2015.

Agudo-Padrón, A. I.; Bleicker, M. S. First registration of wild rocky coast mussels Perna perna (Linnaeus, 1758) infested by boring bivalves in the "Moleques do Sul" Island, Serra do Tabuleiro Ecological State Park, Palhoça Municipal District, Santa Catarina State, SC, Southern Brazil. FMCS Newsletter Ellipsaria, v. 11, p. 14-15, 2009c. Disponible en: $<$ http://molluskconservation.org/EVENTS/ ELLIPSARIA/Ellipsaria2009_113.pdf $>$. Consultado el 15 abr. 2015.

Agudo-Padrón, A. I.; Bleicker, M. S. Additional new records on continental and marine molluscs of Santa Catarina State, SC, Southern Brazil Region. FMCS Newsletter Ellipsaria, v. 13, p. 20-26, 2011. Disponible en: <http://molluskconservation.org/EVENTS/ ELLIPSARIA/EllipsariaMarch2011.pdf>. Consultado el 15 abr. 2015.

Agudo-Padrón, A. I.; Bleicker, M. S. Acréscimos ao cadastro de moluscos marinhos recentes com ocorrência no Estado de Santa Catarina, Região Central Sul do Brasil. Boletín de la Asociación Argentina de Malacologia, v. 4, p. 14-15, 2014. Disponible en: <http://www.malacoargentina.com.ar/images/ stories/documentos/Boletin_2014-1.pdf>. Consultado el 15 abr. 2015.

Agudo-Padrón, A. I.; Bleicker, M. S.; Saalfeld, K. Recent marine molluscs of Santa Catarina State, SC, Southern Brazil Region: a comprehensive synthesis and check list. VISAYA Net, v. 24, p. 1-17, 2009. Disponible en: <http://www.conchology.be/?t=702\& year= $2009 \&$ volume $=24 \&$ vnet $=1283>$. Consultado el 15 abr. 2015.

Agudo-Padrón, A. I.; Veado, R. V. ad-V.; Saalfeld, K. Moluscos e Saúde Pública em Santa Catarina: subsídios para a formulação estadual de políticas preventivas sanitaristas. Duque de Caxias, RJ: Espaço Científico Livre Projetos Editoriais, 2013. Disponible en: $<$ http://issuu.com/espacocientificolivre/docs/ moluscosesaudepublicaemsantacatarina $>$. Consultado el 15 abr. 2015.

CdB - Conquiliologistas do Brasil. (2010). Espécies brasileiras. Disponible en: $<$ http://www.conchasbrasil.org.br/ conquiliologia/>. Consultado el 13 mar. 2010.

Garcia, F. J. G.; Álvarez, M. D.; Troncoso, J. S. Opistobranquios de Brasil: descripción y distribución de Opistobranquios del litoral de 
Brasil y del Archipiélago Fernando de Noronha. Vigo, España: Feito/Universidad de Vigo, 2008.

Melo, C. M. R.; Silva, F. C.; Gomes, C. H. A. M.; Sole-Cava, A. M.; Lazoski, C. Crassostrea gigas in natural oyster banks in Southern Brazil. Biological Invasions, v. 12, p. 441-449, 2010. Disponible en: $<$ https://docs.google.com/file/d/16BwpyTjmL8o zttEIcEbe-A3b_Q7GFtGLhTURRSItumiC2z VjPFzp7k2UJJnF/edit>. Consultado el 15 abr. 2015.

Oliveira, M. P.; Almeida, M. N. Inventário preliminar dos moluscos do Estado de Minas Gerais, Brasil. Strombus, v. 6, p. 1-6, 2000.

Oliveira, M. P.; Castro, G. A. Adenda ao Ensaio de Catálogo de Moluscos do Brasil de Frederico Lange de Morretes. Boletim do Instituto de Ciências Biológicas e Geociências, v. 26, p. 1-9, 1979.
Ribeiro-Costa, C. S.; Marinoni, L. Invertebrados: Manual de aulas práticas. Ribeirão Preto, SP: Holos Editora, 2006.

Rios, E. C. Compendium of Brazilian sea shells. Rio Grande, RS: Evangraf, 2009.

Thomé, J. W.; Gil, G.; Bergonci, P. E. A.; Tarasconi, J. C. As conchas das nossas praias. Porto Alegre, RS: Redes Editora, 2010.

Wiggers, F.; Veitenheimer-Mendes, I. First record and new combination of a rissoid gastropod from off south Brazil. Biotemas, v. 22, p. 251-254, 2009. Disponible en: <https://periodicos.ufsc.br/index.php/biotemas/ article/view/2175-7925.2009v22n4p251/ 17742>. Consultado el 15 abr. 2015.

Información de licencia: Este es un artículo de acceso abierto distribuido bajo los términos de la licencia Creative Commons Attribution License, que permite el uso ilimitado, distribución y reproducción en cualquier medio, siempre que la obra original esté debidamente citados. 